\title{
Simple Patch Antenna with Filtering Function Using Two U-Slots
}

\author{
Youngje Sung*
}

\begin{abstract}
In this study, two U-slots of different sizes are used to combine the filtering function with a patch antenna. The U-shaped slots are etched into the patch, and currents in the opposite direction exist around these slots. Therefore, the currents cancel each other out, and a radiation null is formed. As a result, two radiation nulls are implemented on the left and right sides of the passband. To demonstrate the novelty of the proposed concept, a filtering patch antenna with a center frequency of $3.21 \mathrm{GHz}$ and a $10 \mathrm{~dB}$ impedance bandwidth of $19.9 \%$ is designed and fabricated. High suppression levels of 25.33 and $19.32 \mathrm{~dB}$ in the lower and higher stopbands, respectively, are achieved. Therefore, a sharp band skirt and good selectivity are exhibited in the boresight gain response. The two radiation nulls are located at 2.4 and $3.7 \mathrm{GHz}$ and can be independently adjusted.
\end{abstract}

Key Words: Filtering Antenna, U-Slot Antenna.

\section{INTRODUCTION}

The operating frequency band of an antenna is generally defined as $S_{11}$, which is less than $-10 \mathrm{~dB}$. However, $S_{11}$ is only an amount of reflected power, and there is a limitation that does not mirror the radiation characteristics. An important aspect of antenna performance is the gain versus frequency characteristic. However, from the viewpoint of the gain versus frequency characteristic, it is difficult to define the operating frequency band because the gain relative to the frequency is presented as a gentle curve. In a conventional wireless communication system, a bandpass filter (BPF) is placed behind the antenna to support the appropriate bandwidth required by the system [1]. Conversely, the filtering antenna has the advantage of operating as both an antenna and a filter using a single resonator [2].

A single-feed filtering antenna with U-slot has been previ- ously proposed. Table 1 summarizes these structures. In [3], the radiation null characteristics were realized by applying U- and Ishaped strips to the back of the co-planar waveguide feed structure. In this structure, the suppression level of the radiation null exhibited poor performance of approximately $-7 \mathrm{~dB}$ and $-11 \mathrm{~dB}$, and the antenna gain was significantly changed in the operating band. In [4] and [5], two radiation null characteristics were implemented by applying stubs of different lengths to the feed structure. Two radiation nulls were formed by applying two pairs of slits of differing lengths to the patch [6]. The concept of generating a radiation null below the passband by applying a single U-slot to the patch was proposed in $[7,8]$. However, it was not sufficient for obtaining a perfect filtering function with only one radiation null. To create another radiation null, a Ushaped strip was applied to the back of the substrate in [7], and a stack structure and via were used in [8].

Manuscript received January 5, 2021 ; Revised April 17, 2021 ; Accepted May 18, 2021. (ID No. 20210105-007J)

Department of Electronic Engineering, Kyonggi University, Suwon, Korea.

"Corresponding Author: Youngje Sung (e-mail: yjsung@kgu.ac.kr)

This is an Open-Access article distributed under the terms of the Creative Commons Attribution Non-Commercial License (http://creativecommons.org/licenses/by-nc/4.0) which permits unrestricted non-commercial use, distribution, and reproduction in any medium, provided the original work is properly cited.

(c) Copyright The Korean Institute of Electromagnetic Engineering and Science. 
Table 1. Comparison with previous filtering antennas with U-slot

\begin{tabular}{lcccccc}
\hline Study & Freq. (Hz) & BW (\%) & Null level $(\mathrm{dB})$ & Size & Gain $(\mathrm{dBi})$ & Filtering structure \\
\hline Wang et al. [3] & 2.4 & 7 & $-26,-30$ & $\mathrm{r}=0.13 \lambda_{0}, \mathrm{~h}=0.1 \lambda_{0}$ & 4 & Two stubs \\
Hu et al. [4] & 3.5 & 71.7 & $-7,-11$ & $1.1 \lambda_{0} \times 0.88 \lambda_{0} \times 0.145 \lambda_{0}$ & 4.2 & Two U-slots, two U-strips \\
Zhang et al. [5] & 1.9 & 5 & $-20,-40$ & $0.63 \lambda_{0} \times 0.51 \lambda_{0} \times 0.01 \lambda_{0}$ & 6.7 & Two U-shaped patches, multi-stub feedline \\
& 2.6 & 7 & $-30,-40$ & & 7.3 & Two slits \\
Jin et al. [6] & 5.2 & 7 & $-30,-35$ & $0.35 \lambda_{0} \times 0.24 \lambda_{0} \times 0.027 \lambda_{0}$ & 6.6 & Split-ring slot, U-shaped strip \\
Yang et al. [7] & 5 & 19.7 & $-40,-25$ & $0.66 \lambda_{0} \times 0.66 \lambda_{0} \times 0.098 \lambda_{0}$ & 7.4 & U-slot, stack \\
Zhang et al. [8] & 2.5 & 16 & $-24,-24,-26$ & $0.55 \lambda_{0} \times 0.55 \lambda_{0} \times 0.09 \lambda_{0}$ & 9.7 & \\
\hline
\end{tabular}

In this study, two U-slots of different sizes are applied to the patch surface. To implement the two slots on one patch surface, their directions are set differently so that a small U-slot is located within a large U-slot. The two U-slots form radiation nulls at the bottom and top of the passband. The proposed structure has the advantage that both radiation nulls can be independently controlled.

\section{GEOMETRY}

Fig. 1 shows the proposed filtering antenna, where two Ushaped slots are applied to a rectangular patch of size $L_{p} \times W_{p}$. This antenna is located on the top layer of the substrate and the metal surface existing on the opposite layer is removed. The substrate has a dielectric constant of 2.2 and a thickness of 1.57 $\mathrm{mm}$. To improve the antenna bandwidth, the distance between the substrate and ground plane, denoted by $H$, is filled with air.

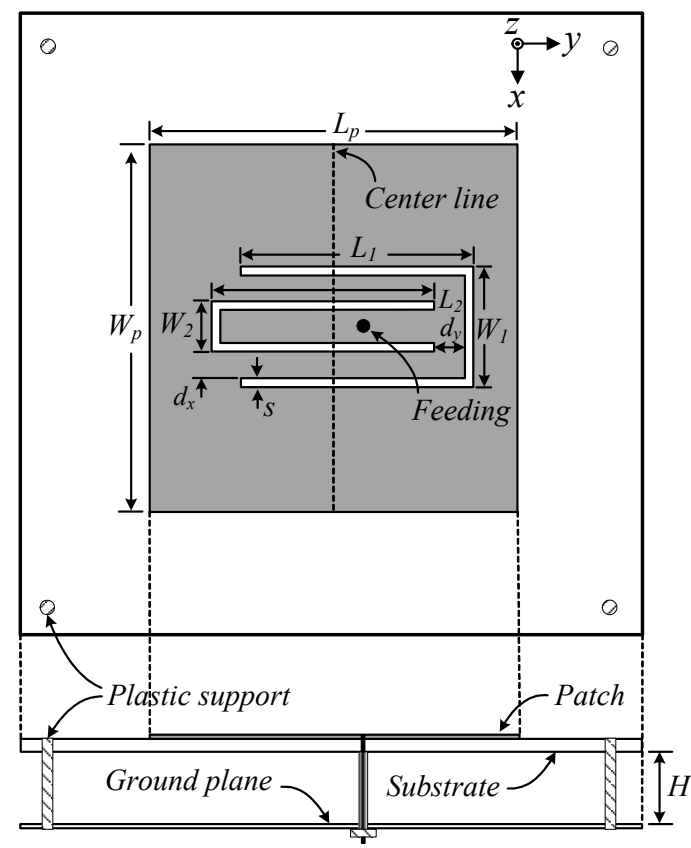

Fig. 1. Proposed antenna with filtering function.
The size of the ground plane is set to $70 \mathrm{~mm} \times 70 \mathrm{~mm}$. The size of the outer U-shaped slot is $L_{1} \times W_{1}$, that of the inner Ushaped slot is $L_{2} \times W_{2}$, and the width of the two slots is set equally to $s$. The distance between the two slots is set to $d_{x}$ and $d_{y}$ in the $x$ - and $y$-axis directions, respectively. The feed point is set $2 \mathrm{~mm}$ away from the patch center. The optimum feed point is obtained using High-Frequency Structure Simulator (HFSS; Ansys Inc., Canonsburg, PA, USA).

\section{OPERATING PRINCIPLE}

Fig. 2(a) and (b) present the simulated results of comparing the reflection coefficient and the realized gain of the four antennas; this result can be used to explain the operating principle of the proposed structure. For the simulation, the dimensions in Fig. 1 are set as $L_{p}=33 \mathrm{~mm}, W_{p}=31 \mathrm{~mm}, L_{1}=18.6 \mathrm{~mm}, W_{1}=$ $8.4 \mathrm{~mm}, L_{2}=17.1 \mathrm{~mm}, W_{2}=4.2 \mathrm{~mm}, s=0.3 \mathrm{~mm}, d_{x}=1.8 \mathrm{~mm}$, $d_{y}=3.9 \mathrm{~mm}$, and $H=8 \mathrm{~mm}$. In Fig. 2, "Ant.with inner slot" indicates a structure that includes only the inner slot in the proposed structure, while "Ant.with outer slot" indicates a structure in which only the outer slot is applied to a rectangular patch. To ensure appropriate comparison, all four antennas are assigned the same parameters. In the case of an antenna with only the inner slot, the matching characteristic deteriorates, which can be improved by adjusting the feed position or slot parameter. The proposed antenna comprises three resonances, as shown in Fig. 2(a). The antenna with only the outer slot has the first and second resonances among the three resonances, whereas that with only the inner slot has the second and third resonances. This shows that the outer slot forms the first resonance, while the inner slot forms the third resonance. Furthermore, the second resonance frequency is formed by the patch.

A rectangular patch without two slots exhibits a single resonance characteristic and has the highest gain at the resonance frequency but does not have a radiation null frequency characteristic on the left and right sides of the passband. Conversely, 


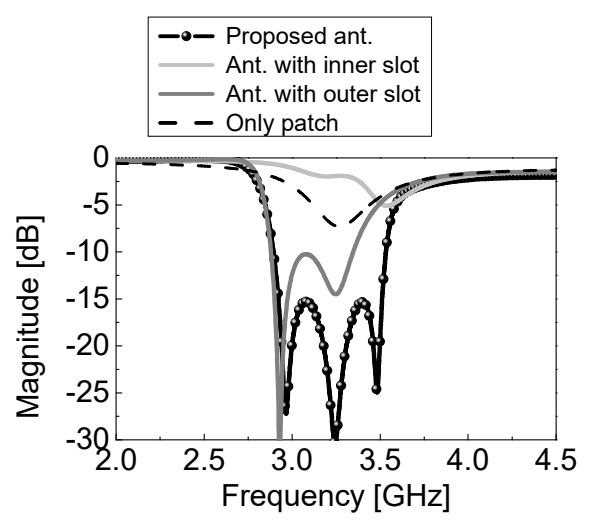

(a)

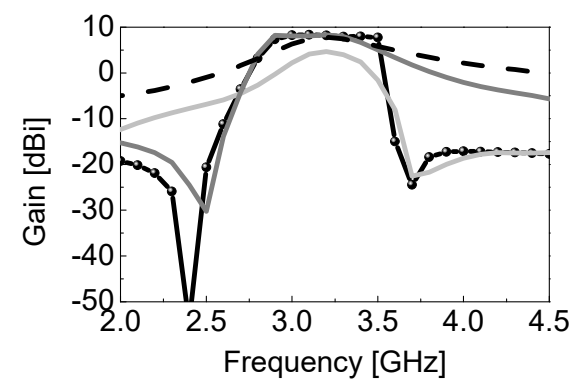

(b)
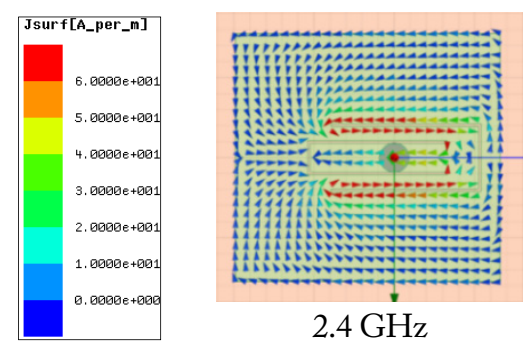

$2.4 \mathrm{GHz}$

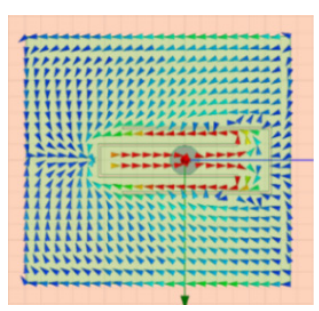

$3.7 \mathrm{GHz}$

(c)
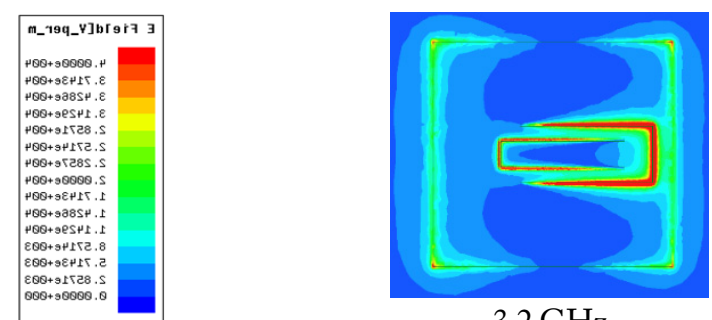

$3.2 \mathrm{GHz}$

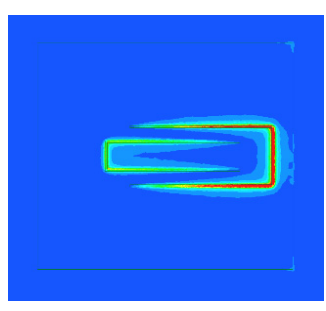

$2.4 \mathrm{GHz}$

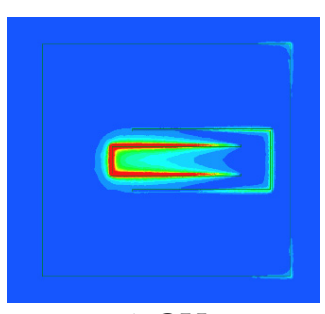

$3.7 \mathrm{GHz}$ (d)

Fig. 2. Comparison of the simulated frequency response of antennas with and without two slots. (a) Reflection coefficient. (b) Realized gain. (c) Surface current distribution on the patch at the two radiation null frequencies. (d) E-field distribution of the patch at the operating frequency and two radiation null frequencies. the proposed structure operates as a filtering antenna because radiation null frequencies exist on both sides of the passband. As shown in Fig. 2(b), the radiation null is formed by the outer and inner slots at frequencies below and above the passband, respectively.

Fig. 2(c) shows the vector current distribution formed at the patch surface. Simulations are performed at two radiation null frequencies, 2.4 and $3.7 \mathrm{GHz}$. Here, the red area indicates that the current is strong, while the blue area indicates that the current is weak. The current is strongly formed along the outer and inner slots at 2.4 and $3.7 \mathrm{GHz}$, respectively. This is consistent with the analysis result, according to which the radiation null is formed by the outer slot at $2.4 \mathrm{GHz}$ and by the inner slot at 3.7 GHz. In Fig. 3, the current directions with respect to the slots are opposite to each other. The currents in different directions that are in proximity cancel each other out. Therefore, Fig. 2(c) proves that the radiation null is caused by the slot. Fig. 2(d) shows the electric field distribution at the operating frequency of the antenna and two radiation null frequencies. At $3.2 \mathrm{GHz}$, a strong electric field is formed on the left and right sides of the patch, and the radiation is found to occur in this area. In addition, strong electric fields are formed in the outer U-slot and inner U-slot at 2.4 and $3.7 \mathrm{GHz}$, respectively, where a radiation null is generated. This supports the fact that the U-slot produces a radiation null.
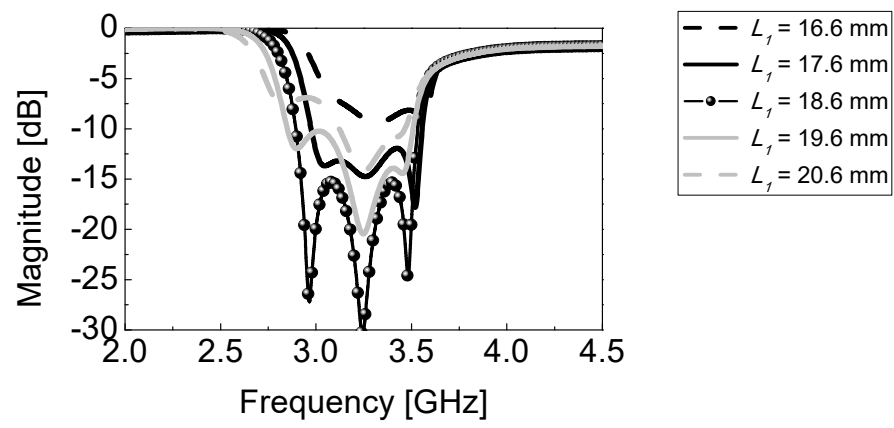

(a)
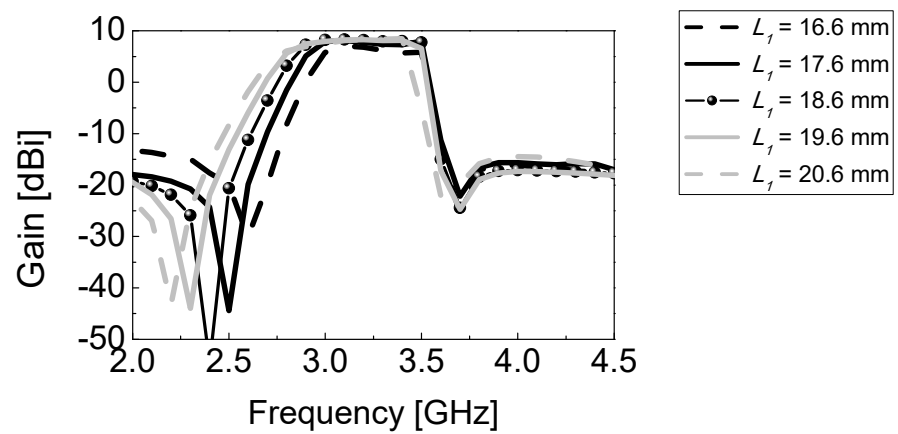

(b)

Fig. 3. Simulated frequency responses of the proposed antenna with different lengths $L_{1}$ : (a) reflection coefficient and (b) gain. 


\section{PARAMETER STUDY}

Fig. 3 shows the simulation results of the reflection coefficient and the realized gain characteristics as the length $L_{1}$ of the outer slot is changed. The parameters set for the simulation are the same as those mentioned previously, except for $L_{1}$. As $L_{1}$ changes, there are two changes in terms of the reflection coefficient. First, as $L_{1}$ increases, the first resonance frequency decreases, but the other two frequencies remain almost constant. This is because the first resonance frequency is formed by the outer slot. Second, as $L_{1}$ changes, the overall matching characteristics of the antenna change rapidly. In the present study, $L_{1}$ is selected as $18.6 \mathrm{~mm}$ from the viewpoint of matching characteristics. The role of the outer slot becomes clear when the gain curve is observed as $L_{1}$ changes. As $L_{1}$ increases, the frequency of the radiation null formed under the passband decreases. At this time, the gain in the passband changes slightly according to the matching characteristics, and the radiation null formed above the passband barely changes.

Fig. 4 presents the simulation results of the antenna characteristics as the inner slot length $L_{2}$ is changed. As $L_{2}$ increases, the third resonance frequency decreases, while the other two resonance frequencies remain constant. Considering the matching characteristics, it is optimal when $L_{2}$ is $17.4 \mathrm{~mm}$. Addition-
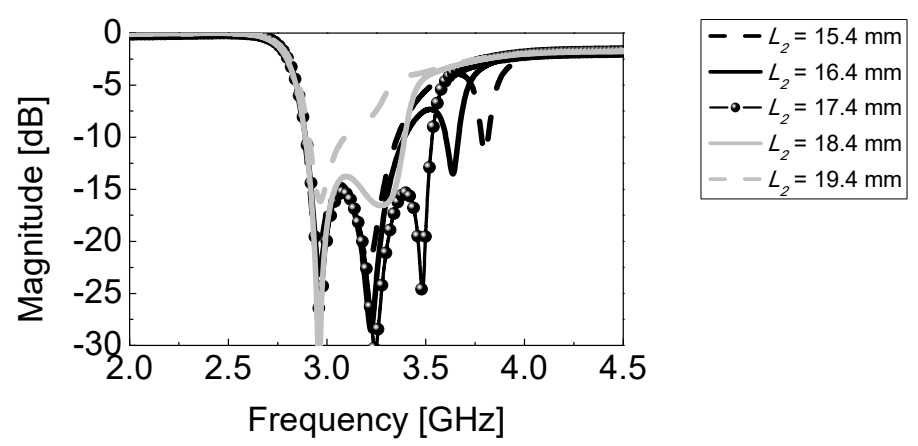

(a)

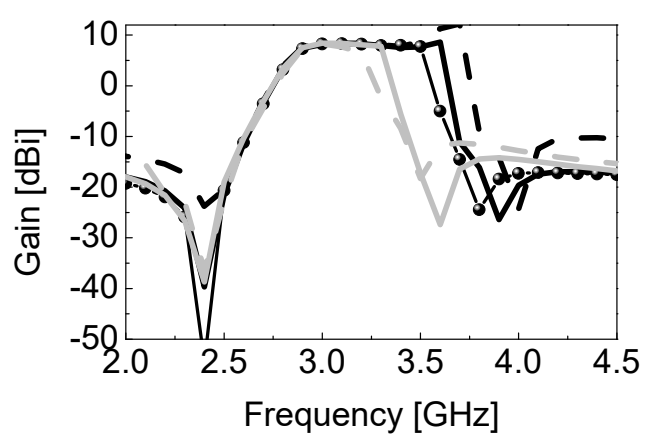

(b)

Fig. 4. Simulated frequency responses of the proposed antenna with different lengths $L_{2}$ : (a) reflection coefficient and (b) gain.

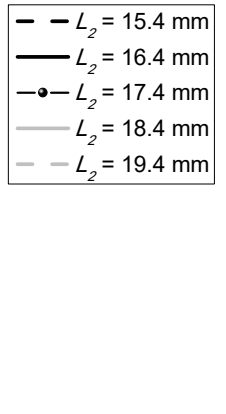

ally, as $L_{2}$ increases, the radiation null frequency located above the passband tends to decrease. The radiation null frequency below the passband does not change with the change in $L_{2}$. However, the radiation suppression level at the corresponding frequency shows a significant difference from $-50 \mathrm{~dB}$ to -20 $\mathrm{dB}$. This is because the inner slot is located between the feed point and the outer slot, and the change in the inner slot affects the radiation null at the frequency below the passband.

\section{SimUlation AND MEASURED RESUlTS}

Fig. 5 shows the simulated and measured frequency responses of the fabricated antenna. The parameters of the fabricated antenna are as follows: $L_{p}=33 \mathrm{~mm}, W_{p}=31 \mathrm{~mm}, L_{1}=18.6 \mathrm{~mm}$, $W_{1}=8.4 \mathrm{~mm}, L_{2}=17.1 \mathrm{~mm}, W_{2}=4.2 \mathrm{~mm}, s=0.3 \mathrm{~mm}, d_{x}=$ $1.8 \mathrm{~mm}, d_{y}=3.9 \mathrm{~mm}$, and $H=8 \mathrm{~mm}$. The proposed structure comprises three resonances and has a wide bandwidth of $19.9 \%$ (2.894-3.533 GHz) using an air substrate. In addition, the measured gain of the antenna is $7.2 \mathrm{dBi}$, with only a small change of $0.2 \mathrm{~dB}$; thus, it exhibits almost constant characteristics in the operating band. Fig. 6 compares the simulated and measured radiation patterns at the center frequency of the antenna (i.e., $3.21 \mathrm{GHz}$ ). Fig. 7 shows a photograph of the fabricated antenna.

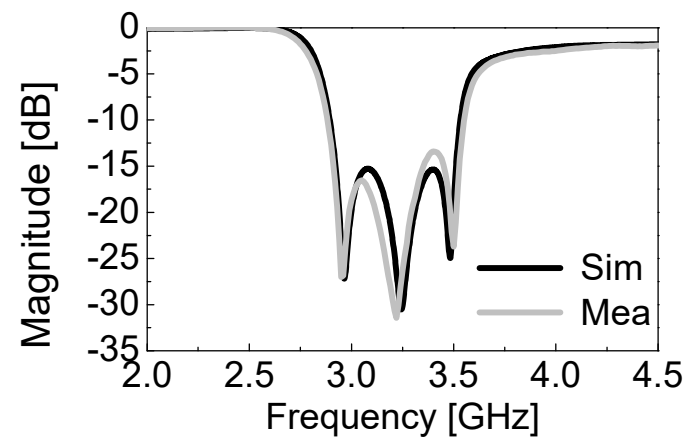

(a)

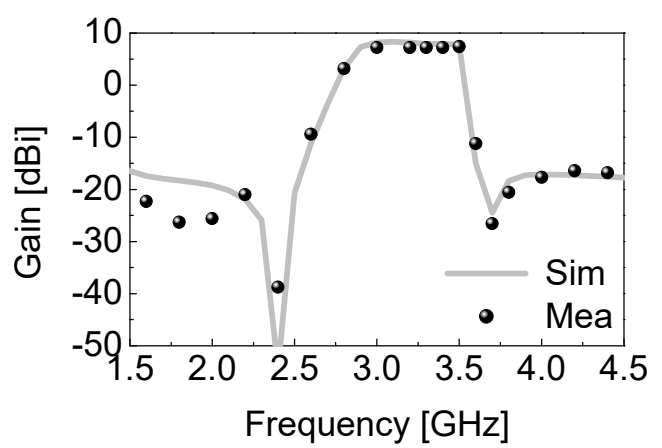

(b)

Fig. 5. Simulated and measured results of the proposed antenna: (a) reflection coefficient and (b) gain. 


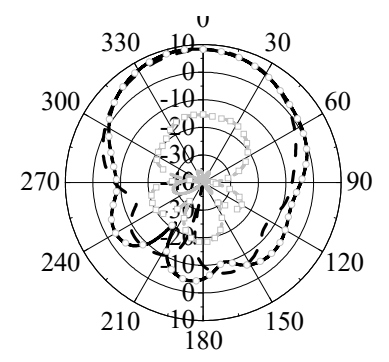

(a)

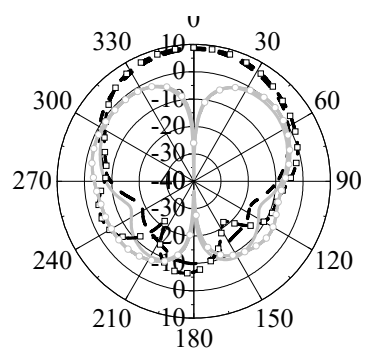

(b)
Fig. 6. Simulated and measured radiation patterns: (a) E-plane and (b) H-plane.

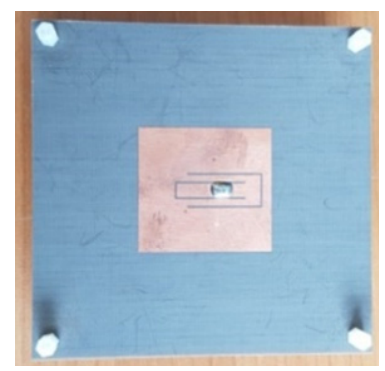

(a)

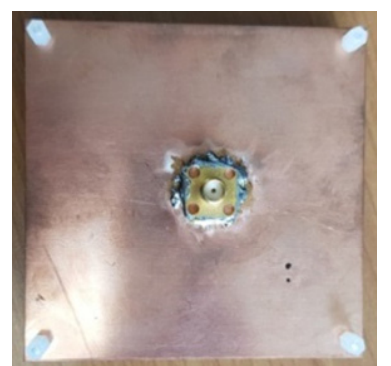

(b)

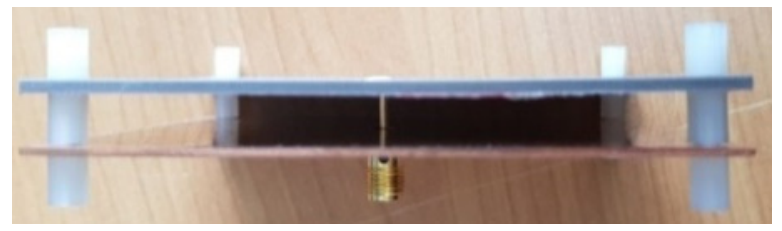

(c)

Fig. 7. Fabricated antenna: (a) top view, (b) bottom view, and (c) side view.

\section{CONCLUSION}

This paper introduces two radiation nulls in the vicinity of the operating band by applying two U-shaped slots to the rectangular patch antenna. The proposed filtering antenna is composed of three resonances, showing a wide bandwidth characteristic and stable and flat gain characteristics in the operating band. In addition, the stopband exhibits a high suppression level exceeding $19 \mathrm{~dB}$ due to a radiation null, indicating high selectivity characteristics. Through the parameter study and simulated current distribution, the operational principle and design strategy of the proposed concept are briefly presented. The demonstrated technique is useful for removing BPFs at the back of the antennas; hence, it can reduce the volume and price of the hardware stage in the communication system.

This work was supported by the Kyonggi University Research Grant 2020.

\section{REFERENCES}

[1] D. M. Pozar, Microwave Engineering. New York, NY: John Wiley \& Sons, 1998.

[2] Y. J. Sung, "Microstrip resonator doubling as a filter and as an antenna," IEEE Antennas and Wireless Propagation Letters, vol. 9, pp. 467-470, 2010.

[3] W. Wang, X. Liu, Y. Wu, and Y. Liu, "A broadband filtering patch antenna using $\mathrm{T}$-probe, transverse stubs, and USlots," IEEE Access, vol. 7, pp. 7502-7509, 2019.

[4] P. F. Hu, Y. M. Pan, X. Y. Zhang, and B. J. Hu, "A compact quasi-isotropic dielectric resonator antenna with filtering response," IEEE Transactions on Antennas and Propagation, vol. 67, no. 2, pp. 1294-1299, 2019.

[5] X. Y. Zhang, Y. Zhang, Y. M. Pan, and W. Duan, "Lowprofile dual-band filtering patch antenna and its application to LTE MIMO system," IEEE Transactions on Antennas and Propagation, vol. 65, no. 1, pp. 103-113, 2017.

[6] J. Y. Jin, S. Liao, and Q. Xue, "Design of filtering-radiating patch antennas with tunable radiation nulls for high selectivity," IEEE Transactions on Antennas and Propagation, vol. 66, no. 4, pp. 2125-2130, 2018.

[7] W. Yang, Y. Zhang, W. Che, M. Xun, Q.Xue, G. Shen, and W. Feng, "A simple, compact filtering patch antenna based on mode analysis with wide out-of-band suppression," IEEE Transactions on Antennas and Propagation, vol. 67, no. 10, pp. 6244-6253, 2019.

[8] X. Y. Zhang, W. Duan, and Y. M. Pan, "High-gain filtering patch antenna without extra circuit," IEEE Transactions on Antennas and Propagation, vol. 63, no. 12, pp. 5883-5888, 2015.

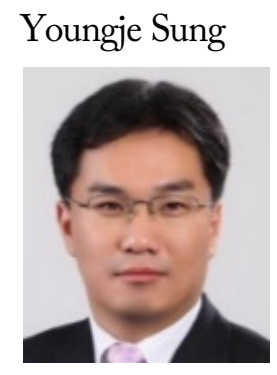

was born in Incheon, Korea, in 1975. He received his B.S., M.S., and Ph.D. degrees from Korea University, Seoul, Korea, in 2000, 2002, and 2005, respectively. From 2005 to 2008, he was a senior engineer with the Antenna R\&D Laboratory, Mobile Phone Division, Samsung Electronics, Korea. In 2008, he joined the Department of Electronic Engineering, Kyonggi University, Suwon, Korea, where he is currently a professor. His research interests include reconfigurable antennas, cellphone antennas, wideband slot antennas, multifunction devices, compact circular polarized antennas, and compact dual-mode filters. Prof. Sung is currently serving as a reviewer for the IEEE Transactions on Microwave Theory And Techniques, IEEE Transactions on Antennas and Propagation, IEEE Microwave and Wireless Components Letters, IEEE Antennas and Wireless Propagation Letters, Progress in Electromagnetic Research, IET Electronics Letters, IET Microwaves, Antennas and Propagation. 